\title{
The Evaluation of Functional Mobility Chronic Hemiparetics Submitted to Group Physiotherapy in the Training Circuit Format
}

\author{
Carvalho AC ${ }^{*}$, Silva FA, Mathias MB, Bofi TC, Barbatto LM, Broek VNVD, Lorenconi RMR and Oliveira \\ MCS
}

Physiotherapy Department, Universidade Estadual Paulista - UNESP - Presidente Prudente Campus, Brazil

${ }^{*}$ Corresponding author: Carvalho AC, Physiotherapy Department, Universidade Estadual Paulista-UNESP-

Presidente Prudente Campus, Brazil, Tel: +55 18 32295800, E-mail: augustocesinando@gmail.com

Citation: Carvalho AC, Silva FA, Mathias MB, Bofi TC, Barbatto LM, et al. (2017) The Evaluation of Functional Mobility Chronic Hemiparetics Submitted to Group Physiotherapy in the Training Circuit

Format. J Neurol Neurol Disord 3(2): 203. doi: 10.15744/2454-4981.3.203

Received Date: February 16, 2017 Accepted Date: June 07, 2017 Published Date: June 12, 2017

\begin{abstract}
Objective: To evaluate the functional mobility of chronic hemiparesis sufferers who undergo 12 weeks of GPCT.

Method: We selected 10 chronic hemiparetic patients, aged 21 or over with a medical referral and one-sided hemiparesis with an injury time of $\geq 12$ months, capable of walking with or without help and having the ability to perform walking tests. Interviews to collect personal data were conducted, which were then evaluated by Time up \& go (TUG) which recorded the time taken for the patient to stand up from a chair, walk 3 meters, return and sit constituting the initial assessment (AV1) and after 12 weeks of intervention with GPCT the final evaluations were taken (AV2).

Results: Statistical analysis showed that the values of AV1 TUG $(0.25 \pm 0.40 \mathrm{~m} / \mathrm{s})$ do not show significant differences in the values of the AV2 $(0.27 \pm 0.44 \mathrm{~m} / \mathrm{s})$. The patients did not show improvement in functional mobility and with this it can be inferred that GPCT did not alter mobility; but patients had the opportunity to maintain their functional abilities by avoiding a sedentary lifestyle, and its consequences, since these patients could practice exercise weekly. The time and intensity of treatment should be studied.

Conclusion: The therapeutic protocol and time used did not modify the functional mobility of chronic hemiparesis sufferers submitted to GPCT.

Keywords: Stroke; Hemiplegia; Circuit-Based Exercise; Physical Therapy Specialty
\end{abstract}

\section{Introduction}

Stroke is a chronic neurological disease that causes different types of disability in affected patients, from requiring assistance to being totally dependent on caregivers to perform activities of daily living such as showering, toileting, and dressing [1-3]. The hemiparesis is a more common disability of stroke with loss of strength and poor motor control in upper-extremity and lowerextremity pareses, capable of causing the restriction of movement of the joints and also muscle contraction capacity, which in turn changes functionality [1]. In addition, motor impairment triggers bodily imbalance, increases resistance to walking and the fear of falling, leading to a decrease of physical activity, leading to a sedentary lifestyle [1,4-6].

Hemiparesis causes a change to a patients gait because they cannot effectively use the ground reaction force of the non-paretic side, resulting in decreased stride length in a complete cycle of walking, making it slower and generating an inefficient pattern that requires increased power consumption while walking [7].

Any rehabilitation program directed to these patients must contain sensory motor stimuli that are effective enough to assist in the reorganization of the brain by performing active exercises that mimic tasks of daily living and self-care, plus be performed in a social and familiar environment [8,9]. Therefore, rehabilitation is very important as it aids in the recovery of functional skills and maintains cardiovascular and metabolic health [10].

Although it was found that the greatest recovery of function occurs in the first 3 months after stroke, there is evidence demonstrating that individuals can improve performance in functional tasks and aerobic capacity, even those in a chronic condition [7,11-13].

Physical exercises are being recommended as a component of rehabilitation programs for post stroke patients in order to prevent 
adverse effects and improve function [1]. Based on this assumption, some types of rehabilitation programs enter patients into individual sessions of conventional physiotherapy as well as training circuit therapy [14]. Both therapies have the potential to improve functional mobility of hemiparetic sufferers, but are employed in different ways. Conventional therapy occurs under the direct and constant supervision of a physical therapist while active, assisted or passive exercises are being performed, in a ratio of one therapist for a patient, while group physiotherapy in a circuit training format (GPCT) emphasizes the presence of a group of patients and different training stations [14,15]. Both therapies seek the development of movement, with an aim of improving performance in carrying out daily activities [16].

During GPCT there are exercises and activities specific to daily tasks, presented in an intensive and active way [15]. The main component of this type of therapy is its realization in a collective environment with more than two participants per therapist, with a focus on the repetitive practice of functional tasks and the continuous progression of the exercises [16]. Although many of the activities have a strength or fitness component, the main focus is on repetitive practice of specific daily motor tasks [14].

GPCT is also able to improve motivation, provide incentives and raise awareness of health and communication between people, leading to greater psychological well-being within the patient [16]. In order to improve the understanding of GPCT, further poststroke trials are required to identify results by assessing the functional motor performance of hemiparetic patients, to contribute to literature findings and guide future care. In view of this, the objective of this study was to evaluate the functional mobility of chronic hemiparetic patients submitted to 12 weeks of GPCT.

\section{Materials and Methods}

In order to carry out this methodological clinical study, data on hemiparetic patients attending physiotherapeutic care at the Center for Studies and Care in Physical Therapy and Rehabilitation of the Faculty of Sciences and Technology of the State University of Paulista, Brazil was used.

Patients with a medical referral and unilateral hemiparesis with a lesion time equal to or greater than 12 months were included in the study. We used patients who were able to walk with or without the aid of bracing, had the ability to perform a walk test and who did not have cognitive deficits when assessed by the Mini-Mental State Examination (cutoff point for illiterate 18/19 and with the School instruction 24/25) [17]. In addition, the change in muscle tone of the paretic ankle extensors had to score different from zero on the Modified Ashworth scale [18]. Subjects with double hemiparesis, injury time less than 12 months, and those with adverse health conditions, such as other non-stroke-related neurological or orthopedic diseases, were not included in the study.

All the volunteers were informed about the objectives and procedures of the study and after agreeing to participate, signed a free and informed consent form. The study was approved by the Institution's Ethics Committee (CAAE: 45076015.3.0000.5402).

An individual interview was conducted to collect the weight, height and verification of inclusion criteria. Before starting GPCT at stations, the patients underwent a personal interview for data collection and then had the initial assessment (AV1) using Time up and go (TUG), which assesses functional mobility and gait [19]. After the first evaluation treatment began with two GPCT sessions per week for a period of 12 weeks. After this period the second evaluation was performed (AV2).

Treatment with GPCT consisted of 10 interconnected spaces called stations with different degrees of difficulty, using sticks, chairs, a ladder and ramp, cones and signalling maps. The activities carried out at each station are shown in Table 1.

\begin{tabular}{|c|c|}
\hline Station number & Description of exercises \\
\hline Station 1 & $\begin{array}{l}\text { Flexion and extension of the hip and knees alternately } \\
\text { supporting the feet on a platform } 50 \text { centimeters high. }\end{array}$ \\
\hline Station 2 & $\begin{array}{l}\text { Hands intertwined with the patient sitting to each side to } \\
\text { touch a wall. }\end{array}$ \\
\hline Station 3 & $\begin{array}{l}\text { Flexion and shoulder extension with resistance using an } \\
\text { elastic band. }\end{array}$ \\
\hline Station 4 & $\begin{array}{l}\text { Flexion and extension of hip, knee and ankle (squatting) } \\
\text { on a } 20 \mathrm{~cm} \text { step. The hemiparetic can support themselves } \\
\text { using a bar. }\end{array}$ \\
\hline Station 5 & Walk -a distance of 5 meters and return in position. \\
\hline Station 6 & $\begin{array}{l}\text { Transfer plastic bottles weighing } 1 \text { kilogram of a shelf } 50 \\
\text { centimeters in high to one } 1.20 \text { centimeters and vice versa. }\end{array}$ \\
\hline Station 7 & $\begin{array}{l}\text { Rotation of trunk and extension and flexion of shoulder } \\
\text { simulating rowing movement. }\end{array}$ \\
\hline Station 8 & $\begin{array}{l}\text { Side walking on a parallel bar with obstacles } 5 \text { centimeters } \\
\text { high, } 30 \text { centimeters apart. }\end{array}$ \\
\hline Station 9 & Climb a ladder, then descend. \\
\hline Station 10 & Go up and down a step, leaning on a bar. \\
\hline
\end{tabular}

Table 1: Description of exercises performed in stations 
At the beginning of each GPCT session blood pressure was measured and cards distributed with the station number that each volunteer would start the session at. The time each volunteer exercised at each station was 3 minutes and at the end of this time, the volunteer passed to the next circuit station. When the patients could not perform the task by themselves the researchers assisted them doing the assigned exercise. A maximum of 2 patients were placed at each station. The duration of each GPCT session was 50 minutes.

For the statistical analysis, Microsoft Excel 2013 was used and the descriptive statistics were presented in the form of means and standard deviations. Intragroup comparisons and inter-analyses were performed via analysis of variance (ANOVA). The Levene test was used to test the homogeneity of the samples in the different groups, and then proceeded with the T-student test to compare the measure outcome from pre to post-treatment. A p value less than 0.05 was considered significant.

\section{Results}

Ten hemiparetic subjects participated, of which 6 were male and 4 female, $59.7 \pm 10.4$ years old. The average time after the stroke was $81 \pm 26.6$ months, and the right hemiparesis was observed in 8 individuals and the left in 2 individuals. The average BMI was $28,04 \pm 7,28 \mathrm{~kg}$ and the average score of Mini-Mental State Examination was $20.4 \pm 2.3$ points.

None of the participants used orthoses, but two of them relied on a walking stick. Three subjects were using beta blockers. Regarding risk factors, all individuals were hypertensive and three had diabetes mellitus and one was a smoker. In the TUG, the volunteers had an average speed of $0.25 \pm 0.40 \mathrm{~m} / \mathrm{s}$ when analyzed in AV1. After 12 weeks of GPCT (AV2) the results were $0.27 \pm 0.44 \mathrm{~m} / \mathrm{s}$. The statistical analysis shows there was no significant difference between AV1 and AV2 ( $\mathrm{p} \leq 0.05)$.

\section{Discussion}

The results demonstrated that this therapy is safe and feasible for chronic hemiparetic patients and corroborates the recommendations of the American Heart Association, which promotes research using innovative methods to increase the duration of functional practice with the least resources; although it has not demonstrated improvement in functional mobility characteristics [20].

The lack of functional improvement may be due to the small impact of the training on walking capacity, and it may also be that the tasks used in the FGCT do not focus on gait specificity because they stimulate not only gains in speed, but generalized functional parameters as well. The results of this study resemble the results of Nelles G et al. (2001), who also did not find functional changes in the hemiparetic patients that completed the FGCT, but the positron emission tomography of these individuals showed changes that indicate cerebral plasticity [21].

The learning of a motor skill, which occurs through a repeated sequence, can improve cortical activation, inducing neuroplastic change, and can remedy patterns of adaptation of cerebral activity after a stroke [22,23]. FGCT is a therapeutic model that involves task-oriented functional practice and uses a functional approach in an intensive way to induce a functional improvement in hemiparetic sufferers. This practice also suggests that training can be organized as a circuit with a series of workstations that offer important improvements in gait, balance and, consequently, daily activities [7,11,14].

The results of this study suggest that the amount of specific practice completed during FGCT may not be sufficient to drive changes was measured with the tests used. However, this model of group therapy has been proposed to increase the amount of time spent on specific tasks in order to improve the functional capacity of hemiparetics [24]. They had the opportunity to maintain their functional abilities, avoiding a sedentary lifestyle and its consequences, since these patients practiced weekly. In addition, it was observed that FGCT is an inexpensive, safe method with high adherence rates and an absence of serious adverse events [20,24].

There is a need for more research on the FGCT model, mainly in the time spent and the optimal delivery, needed to improve functional recovery of a hemiparetic patient vai active tasks, and its relation with the improvement of functional abilities. The results suggest that a circuit-training program is an intervention method with potential to improve the walking ability of hemiparetic individuals, as well as offering the opportunity to perform exercises and decrease sedentary lifestyles with less financial cost to health systems.

\section{Conclusion}

The therapeutic protocol used and the time permitted was not able to modify the functional mobility of chronic hemiparetic patients submitted to GPCT.

\section{References}

1. Kim B, Park Y, Seo Y, Park S, Cho H, et al. (2016) Effects of individualized versus group task-oriented circuit training on balance ability and gait endurance in chronic stroke inpatients. J Phys Ther Sci 28: 1872-5.

2. Polese JC, Tonial A, Fung FK, Mazuco R, Oliveira SG, et al. (2008) Assessment Scales of quality of life in Brazilian patients after Stroke. Ver Neurocienc16:175-8. 3. Port IGL, Wevers LEG, Lindeman E, Kwakkel G (2012) Effects of circuit training as alternative to usual physiotherapy after stroke: randomised controlled trial. BMJ 344:e2672. 
4. Jørgensen JR1, Bech-Pedersen DT, Zeeman P, Sørensen J, Andersen LL, et al. (2010) Effect of intensive outpatient physical training on gait performance and cardiovascular health in people with hemiparesis after stroke. Phys Ther. 90: 527-37.

5. Hornby TG, Straube DS, Kinnaird CR, Holleran CL, Echauz AJ, et al. (2011) Importance of specificity, amount, and intensity of locomotor training to improve ambulatory function in patients poststroke. Top Stroke Rehabil 18: 293-307.

6. Gallanagh S, Quinn TJ, Alexander J, Matthew R Walters MR (2011) Physical Activity in the Prevention and Treatment of Stroke. ISRN Neurol 2011 : 953818.

7. Park KT, Kim HJ (2016) Effect of the a circuit training program using obstacles on the walking and balance abilities of stroke patients. J Phys Ther Sci 28: 1194-8.

8. Langhorne P, Coupar F, Pollock A (2009) Motor recovery after stroke: a systematic review. Lancet Neurol 8: 741-54.

9. Veerbeek JM, Wegen E, Peppen R, Wees PJ, Hendriks E, et al. (2014) What is the evidence for physical therapy poststroke? A systematic review and metaanalysis. Plos One 9: e87987.

10. Hillier S, English C, Crotty M, Segal L, Bernhardt J, et al. (2011) Circuit class or seven-day therapy for increasing intensity of rehabilitation after stroke: protocol of the circuit trial. Int J Stroke 6: 560-5.

11. Dean CM, Richards CL, Malouin F (2008) Task-related circuit training improves performance of locomotor tasks in chronic stroke: A randomized, controlled pilot trial. Arch Phys Med Rehabil 81: 409-17.

12. English C, Hillier SL (2010) Circuit class therapy for improving mobility after stroke. Cochrane Database Sys Rev 7: CD007513.

13. Brazzelli M, Saunders DH, Greig CA, Mead GE (2011) Physical fitness training for stroke patients. Cochrane Database Sys Rev11: CD003316.

14. English CK, Hillier SL, Stiller KR, Warden-Flood A (2007) Circuit class therapy versus individual physiotherapy sessions during inpatient stroke rehabilitation: a controlled trial. Arch Phys Med Rehabil 88: 955-63.

15. English C, Hillier S (2011) Circuit class therapy for improving mobility after stroke: a systematic review. J Rehabil Med 43: 565-71.

16. Song HS, Kim JY, Park SD (2015) Effect of the class and individual applications of task-oriented circuit training on gait ability in patients with chronic stroke. J Phys Ther Sci 27: 187-9.

17. Pinheiro MB, Scianni AA, Ada L, Faria CD, Teixeira-Salmela LF (2014) Reference values and psychometric properties of the lower extremity motor coordination test. Arch Phys Med Rehabil 95:1490-7.

18. Gregson JM, Leathley M, Moore AP, Sharma AK, Smith TL, et al. (1999) Reliability of the Tone Assessment Scale and the modified Ashworth scale as clinical tools for assessing poststroke spasticity. Arch Phys Med Rehabil 80: 1013-6.

19. Miyamoto ST, Lombardi Junior I, Berg KO, Ramos LR, Natour J (2014) Brazilian version of the Berg balance scale. Braz J Med Biol Res 37: $1411-21$.

20. Port IGL, Wevers LEG, Lindeman E, Kwakkel G (2012) Effects of circuit training as alternative to usual physiotherapy after stroke: randomized controlled trial. BMJ 344: e2672.

21. Nelles G, Jentzen W, Jueptner M, Müller S, Diener HC, et al. (2001) Arm training induced brain plasticity in stroke studied with serial positron emission tomography. Neuroimage 13: 1146-54.

22. Boyd LA, Vidoni ED, Wessel BD (2010) Motor learning after stroke: is skill acquisition a prerequisite for contralesional neuroplastic change? Neurosci Lett 482: $21-5$.

23. Kerr AL, Cheng SY, Jones TA (2011) Experience-dependent neural plasticity in the adult damaged brain. J Commun Disord 44: 538 -48.

24. Winstein CJ, Stein J, Arena R, Bates B, Cheney LR, et al. (2016) Guidelines for Adult Stroke Rehabilation and Recovery. Stroke 48 : e1-73.

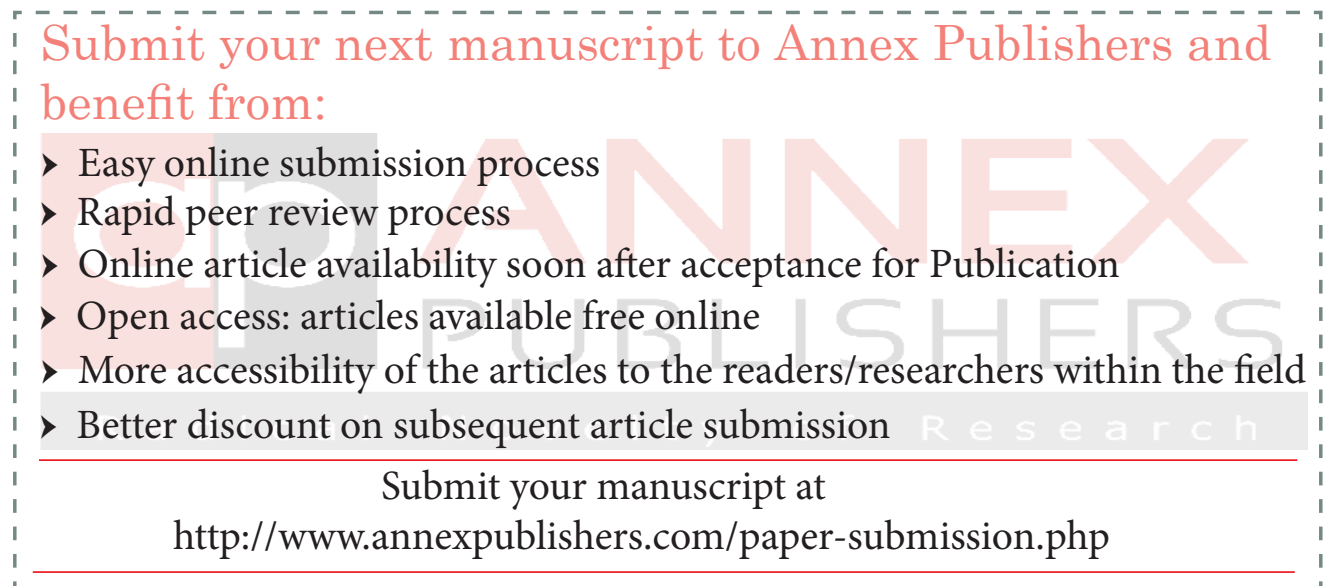

